\title{
Bandwidth Improvement in Large Reflectarrays by Using True-Time Delay
}

\author{
Eduardo Carrasco, Student Member, IEEE, José A. Encinar, Member, IEEE, and Mariano Barba
}

\begin{abstract}
A significant improvement in the bandwidth of large reflectarrays is demonstrated using elements which allow true-time delay. Two identical, large reflectarrays have been designed using different phase distributions to generate a collimated beam. In the former, the phase distribution is truncated to $360^{\circ}$ as is usual in reflectarray antennas, while in the second, the true phase delay is maintained (three cycles of $360^{\circ}$ ). The chosen phase-shifter elements are based on previously measured and validated patches aperture-coupled to delay lines. The radiation patterns for both reflectarrays have been computed at several frequencies and the gain is represented as a function of frequency for both cases. Bandwidth curves are presented as a function of the reflectarray size.
\end{abstract}

Index Terms-Broadband antennas, reflectarrays, time-delay arrays.

\section{INTRODUCTION}

$\mathbf{T}$ HE most severe drawback in reflectarray antennas is their inherent narrow-band performance, and much effort has been made in recent years to overcoming this limitation [1]-[11]. Reflectarray bandwidth is limited mainly by two different factors as described in [1]. The first is the narrow band of the radiating elements; the second is the differential spatial phase delay resulting from the different paths from the feed to each point on the wave front of the radiated beam.

The bandwidth limitation produced by the radiating element is the more stringent for moderate size reflectarrays, as demonstrated in [2]. Different types of reflectarray elements have been proposed to improve the element bandwidth in printed reflectarrays, including stacked rectangular [3], [4] and ring [5] patches, double crossed loops [6], and patches with other shapes [7].

In a recent work, a reflectarray based on artificial impedance surfaces has been proposed to increase the bandwidth of reflectarrays using a single layer of printed elements [8] arranged in a regular lattice with period of less than half a wavelength. This configuration, based on the reduction of the period, allowed increasing the bandwidth up to a $20 \%$ for a reflectarray of around ten wavelengths in diameter. For this antenna diameter, the errors introduced by the differential spatial phase delay do not produce a significant reduction in the bandwidth.

On the other hand, the bandwidth limitation produced by the differential spatial phase delay is critical for electrically large

\footnotetext{
Manuscript received October 29, 2007; revised March 10, 2008. Published August 6, 2008 (projected). This work was supported in part Spanish Ministry of Science and Technology under the project MEC TEC 2004-02155 and by the Mexican National Council of Science and Technology (CONACYT).

The authors are with the Electromagnetism and Circuit Theory Department, Universidad Politecnica de Madrid, 28040 Madrid, Spain (e-mail:carrasco@etc. upm.es).

Digital Object Identifier 10.1109/TAP.2008.927559
}

antennas and small F/D ratios [1], [2]. Usually, the required phase value to compensate for the different paths of the wave from the feed horn to each array element is only achieved at the central frequency within a range of $360^{\circ}$. When frequency varies, a phase error proportional to the differences in path length is produced. This effect produces a small reduction in gain for pencil beam reflectarrays as shown in [2], and produces a significant distortion of the beam shaping in contoured beam reflectarrays [4] when frequency varies out of the central frequency. This band limitation is the same as that which occurs in zoned reflectors [12], in which the depth of a parabolic reflector is reduced in steps of an integer number of half wavelengths, in a similar manner as in zoned dielectric lenses [13]. Although the surface design of an unzoned lens is independent of frequency, zoning the lens reduces its mass and losses in the dielectric material, but limits the bandwidth through the introduction of frequency dependence [14].

Parabolic reflector antennas use the physical geometry to equalize spherical waves arriving from the feed horn to form a planar wave front. This phase equalization is independent of the frequency, and it is equivalent to the true-time delay (TTD) technique, as it is called in phased arrays. In contrast, printed elements on reflectarrays are normally used for phase-shift compensation in a limited $360^{\circ}$ phase range.

A phase-delay range with more than one cycle of $360^{\circ}$ can be used to improve the bandwidth of reflectarrays. Stubs of variable length attached to rectangular patches [15] can introduce a TTD, but with a very restricted room for long lines and important spurious radiation levels. The use of varying-length lines aperture-coupled to patches [10], [11] can reduce the effect of the differential spatial phase delay through the introduction of a physical path that produces TTD in a wide phase range. However, in this type of element, the phase of the total reflected field, including the field reradiated by the patch and the one reflected by the grounded dielectric, exhibits a nonlinear response produced by the resonances of the structure, as shown in [16]. In order to achieve TTD, the elements must be designed to provide a linear phase response, proportional to the length of the line in a large range of phase delay. For example, the resonance effects were compensated in [17] by properly adjusting the length of the aperture, and a reflectarray element was designed to provide a phase directly proportional to the line length.

The improvement of bandwidth using true-time delay phase compensation in reflectarrays is demonstrated in this paper. For this purpose, two pencil-beam reflectarrays have been designed at $9.65 \mathrm{GHz}$ using two different phase distributions implemented by patches aperture-coupled to delay lines. The first distribution is limited to one cycle of $360^{\circ}$ and the second 


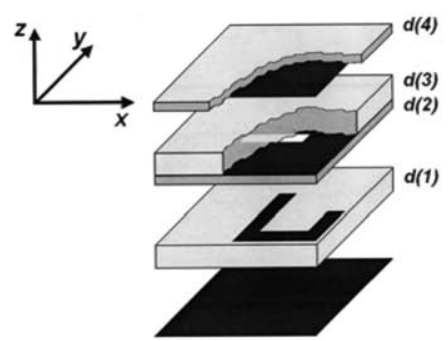

(a)

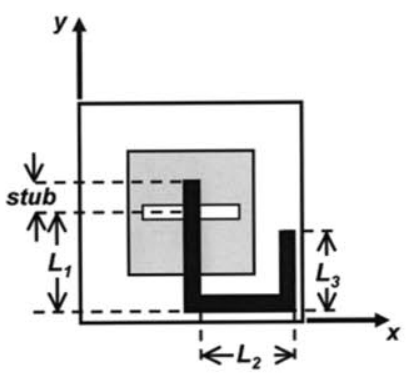

(b)
Fig. 1. Reflectarray element based on patches aperture-coupled to delay lines. (a) Expanded view. (b) Top view.

distribution is expanded up to three times $360^{\circ}$. A bandwidth improvement is demonstrated in the second case, as a result of the TTD.

Additionally, other moderate-sized reflectarrays, which require fewer than three cycles of phase-delay have been designed using limited phase distributions and expanded TTD distributions. The bandwidth curves as a function of the reflectarray size are presented, demonstrating that by using TTD the bandwidth is not reduced in large reflectarrays.

\section{ANalysis, Validation, AND Design OF REFLECTARRAY ELEMENT}

Reflectarray elements based on patches aperture-coupled to delay lines allow a wide range of phase delay if the delay line is bent forming a U-shaped line like that shown in Fig. 1. For the analysis of this type of reflectarray element, a full-wave modular technique described in [18] is used. The element is considered in an infinite array environment and it is analyzed by the method of moments (MoM) in spectral domain by considering an arbitrary incident field on the periodic structure, with its corresponding angle of incidence. The modular approach is based on the individual characterization of each array layer (array of patches, array of apertures, and array of delay lines). First, a generalized scattering matrix (GSM) which characterizes each array layer is computed separately, and then, the total GSM is obtained by the iterative cascading of the individual GSMs of each interface using simple matrix computations. The overall GSM of the cell provides the phase-delay, ohmic losses and cross-polarization produced by the reflectarray element.

For the experimental validation, the well known waveguide simulator (WGS) technique has demonstrated accurate results [19]. A reflectarray element has been designed, manufactured and measured in a WGS for different line lengths. Simulation of an infinite array can be verified by the insertion of some radiating elements inside a waveguide section and measuring the reflection in the excitation port. The imaging action of the waveguide walls simulates the infinite array for one polarization. A WR112 waveguide has been chosen, imposing a unit cell size of $14.25 \mathrm{~mm} \times 12.6 \mathrm{~mm}$ (two radiating elements has been inserted). The geometrical data of the manufactured element are summarized in Table I. The thickness values correspond to that measured in the materials, because sometimes there are discrepancies with respect to nominal values, particularly for Rohacell (layer 3).
TABLE I

DATA FOR THE ElEMENT SimUlated IN WGS

\begin{tabular}{ccrc}
\hline Metal Layer & $\mathrm{X}, \mathrm{mm}$ & \multicolumn{2}{c}{$\mathrm{Y}, \mathrm{mm}$} \\
\hline \hline Line & 1.60 & $\begin{array}{r}2.1(\mathrm{stub})+9.14(\mathrm{~L} 1)+ \\
9.5(\mathrm{~L} 2)+4.9(\mathrm{~L} 3)\end{array}$ \\
\hline Slot & 6.61 & \multicolumn{2}{c}{1.23} \\
\hline Patch & 10.00 & \multicolumn{2}{c}{10.00} \\
\hline Period & 14.25 & \multicolumn{2}{c}{12.60} \\
\hline \hline Dielectric layer & thickness, mm & $\varepsilon_{\mathrm{r}}$ & $\tan \delta$ \\
\hline \hline $\mathrm{d}(1)$ & without ground & --- & --- \\
\hline $\mathrm{d}(2)$ & 0.508 & 3.380 & 0.0050 \\
\hline $\mathrm{d}(3)$ & 1.940 & 1.067 & 0.0002 \\
\hline $\mathrm{d}(4)$ & 0.810 & 3.200 & 0.0030 \\
\hline WR112 section & 26.74 & 1.050 & 0.0002 \\
\hline
\end{tabular}

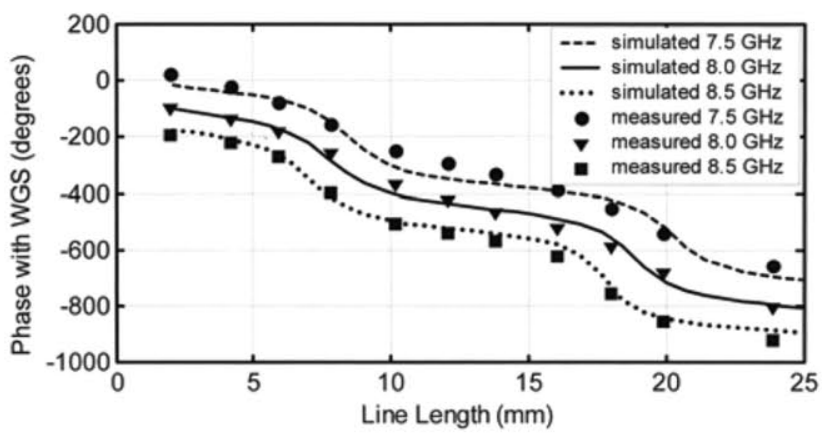

Fig. 2. Simulated and measured phases for reflectarray element in WGS.

TABLE II

DESIgNEd RADIATING ELEMENT DATA

\begin{tabular}{cccc}
\hline Metal Layer & $\mathrm{X}, \mathrm{mm}$ & \multicolumn{2}{c}{ Y, mm } \\
\hline \hline Line & 1.50 & \multicolumn{2}{c}{1.50 (stub) + variable } \\
\hline Slot & 7.00 & \multicolumn{2}{c}{1.00} \\
\hline Patch & 9.30 & \multicolumn{2}{c}{9.30} \\
\hline Period & 16.5 & \multicolumn{2}{c}{16.5} \\
\hline \hline Dielectric layer & thickness, $\mathrm{mm}$ & $\varepsilon_{\mathrm{r}}$ & $\tan \delta$ \\
\hline \hline $\mathrm{d}(1)$ & 7.700 & 1.067 & 0.0002 \\
\hline $\mathrm{d}(2)$ & 0.508 & 3.380 & 0.0050 \\
\hline $\mathrm{d}(3)$ & 2.000 & 1.067 & 0.0002 \\
\hline $\mathrm{d}(4)$ & 0.508 & 3.380 & 0.0050 \\
\hline
\end{tabular}

Fig. 2 shows the comparison between computed and measured phase of the reflection coefficient of the designed element in a frequency band from 7.5 to $8.5 \mathrm{GHz}$. The simulations has been performed using roof-top basis functions to characterize the U-shaped delay line. Straight microstrip lines larger than the waveguide borderline has been measured to produce the phase delay performance. Good agreement between simulations and measurements can be seen with maximum differences in the order of $20^{\circ}$ that is into an acceptable range, mainly produced by photo etching errors, alignment errors, and by the assumption of an infinite array of the delay lines in the simulations.

Once the proposed phase-delay element has been validated, a reflectarray element is designed for a wide range of phase delay. The phase response can be very linear if a proper adjustment of the geometry is performed alleviating the effects produced by the resonances [17]. Depending on the phase values required at the reflectarray elements, the phase-shifter is chosen with a simple $L_{1}$ line segment or with a U-shaped TTD line. The phase and amplitude of the reflection coefficient provided by a reflectarray element with the characteristics of Table II are 


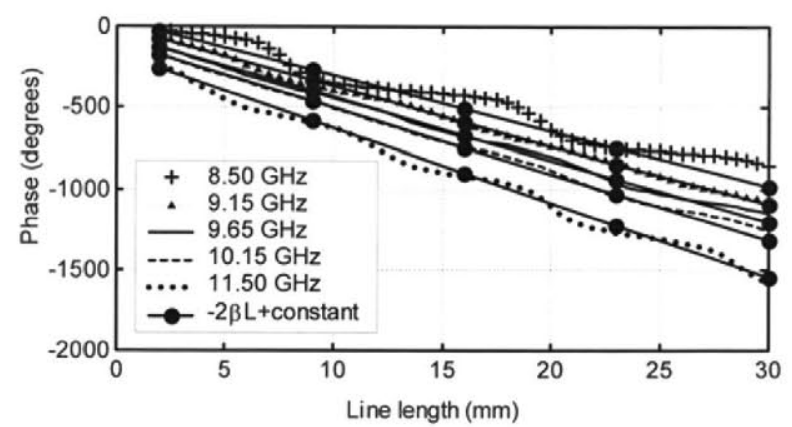

(a)

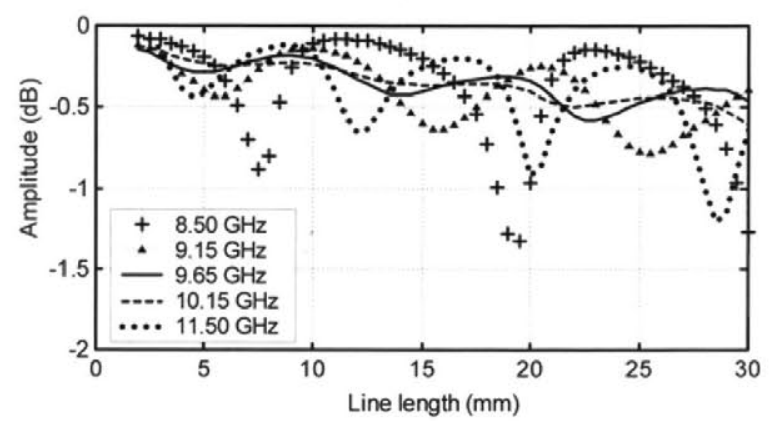

(b)

Fig. 3. Reflection coefficient for reflectarray element as a function of line length for normal incidence, $\theta=0^{\circ}, \varphi=0^{\circ}$. (a) Phase. (b) Amplitude.

shown in Fig. 3 as a function of the line-length for different frequencies when a plane-wave impinges at normal incidence. The phase variation covers the $3 \times 360^{\circ}$ phase range required for the phase-delay distribution and it is compared with an ideal delay line, in which the phase should be $-2 \beta L$, where $\beta$ is the propagation constant at the corresponding frequency and $\mathrm{L}$ the length of the delay line. The only drawback of delay lines is that a longer line produces a higher ohmic loss, as shown in Fig. 3(b). In the present case, the losses have maximum values around 1 $\mathrm{dB}$ at the line resonances. The overall dissipative losses in a reflectarray having patches of all dimensions are expected to be less than $0.5 \mathrm{~dB}$. These losses can be reduced if low-loss materials (as RT-Duroid) are used for layers d(1) and d(4). As the reflection response depends on the incidence angle, Fig. 4 shows the reflection behavior when the wave impinges obliquely with an angle $\theta=30^{\circ}, \varphi=40^{\circ}$. As expected, slight variations in phase and amplitude are produced when the angle of incidence changes. The reflected wave on each element includes the field reradiated directly by the patch, and the field reflected by the structure.

\section{REFLECTARRAY DESIGN}

Considering a reflectarray on the $x-y$ plane illuminated by a feed horn, the required phase distribution, $\Phi\left(x_{i}, y_{i}\right)$, at each element of the array to collimate a beam in the $\left(\theta_{0}, \varphi_{0}\right)$ direction is determined as

$$
\Phi\left(x_{i}, y_{i}\right)=k_{0}\left[d_{i}-\sin \theta_{0}\left(x_{i} \cos \varphi_{0}+y_{i} \sin \varphi_{0}\right)\right]
$$

where $k_{0}$ is the propagation constant in vacuum, $d_{i}$ is the distance from the feed horn to the element $i$ of the array and $\left(x_{i}, y_{i}\right)$

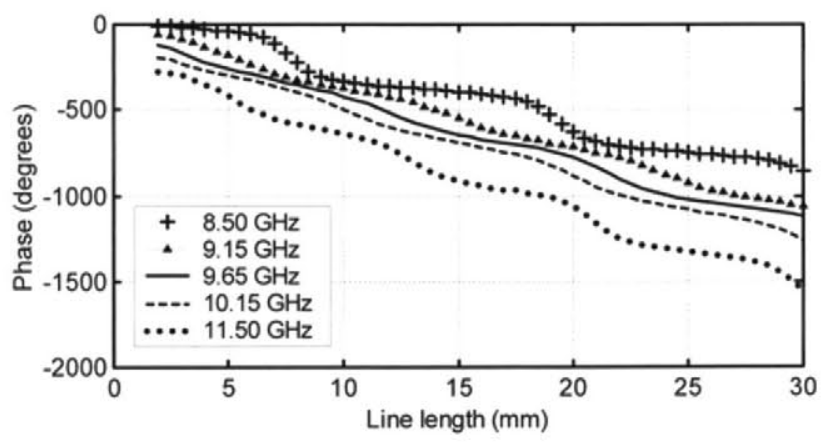

(a)

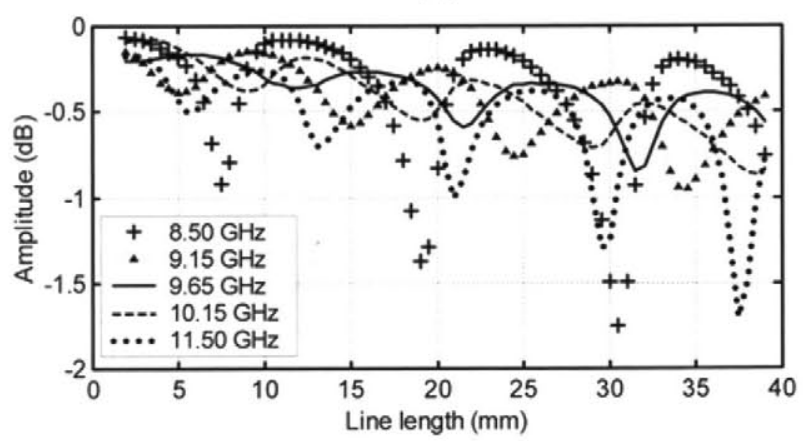

(b)

Fig. 4. Reflection coefficient for reflectarray element as a function of line length for oblique incidence, $\theta=30^{\circ}, \varphi=40^{\circ}$. (a) Phase. (b) Amplitude.

are the coordinates of the element $i$. The phase distribution is usually truncated to a $360^{\circ}$ phase-shift cycle to simplify the implementation of the reflectarray elements. However, the effect of spatial phase delay can be overcome if the phase is expanded to several $360^{\circ}$ cycles.

Fig. 5 shows the phase distribution at $9.65 \mathrm{GHz}$ to produce a pencil beam in the direction $\theta_{0}=18^{\circ} \varphi_{0}=0^{\circ}$ for an elliptic reflectarray antenna made up of 1888 elements arranged in a $50 \times 48$ grid, assuming the feed in the position $X_{F}=$ $-288 \mathrm{~mm}, Y_{F}=0 \mathrm{~mm}, Z_{F}=793 \mathrm{~mm}$, with respect to the reflectarray center in two cases: (a) the phase truncated to $360^{\circ}$ and (b) the phase expanded to three cycles of $360^{\circ}$. The reflectarray element shown in Fig. 1 with the geometrical data summarized in Table II is used to implement the phase distributions.

The design of the reflectarray, for each phase distribution, consists of determining the length of the delay line in each element to achieve the phase distributions represented in Fig. 5. at central frequency. The feed horn is modeled by a $\cos ^{q}(\theta)$ function to determine the level of illumination at each radiating element. Once the reflectarray's geometrical parameters are defined which include aperture, patch, line width and dielectric layers, feed position, and incidence angles, the required phase distribution is computed independently for both cases: phase-shift limited to one $360^{\circ}$ cycle and phase-delay without truncation, see Fig. 5. The reflection coefficient of the impinging wave is calculated at each element localization for an initial line length value. If the phase obtained for this line length is greater than the required phase defined by the phase distribution, the length of the line is incremented with $\Delta L$ and an iterative loop begins until the phase value is less than the required phase. If the phase achieved with the line length is less 


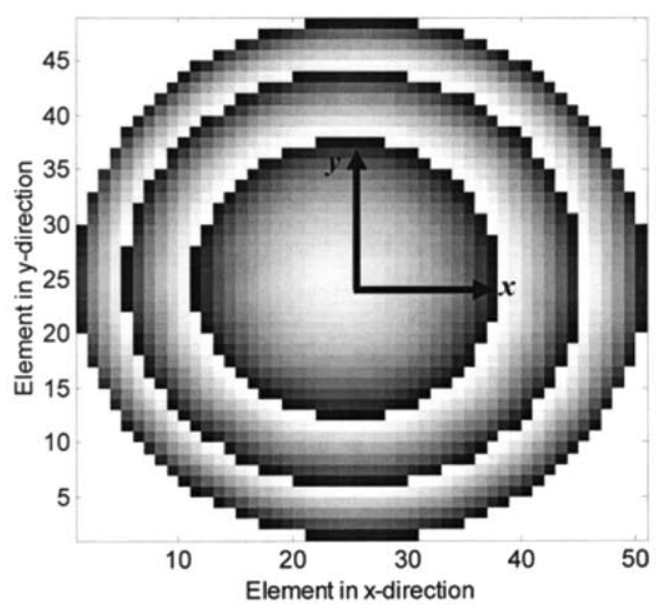

(a)

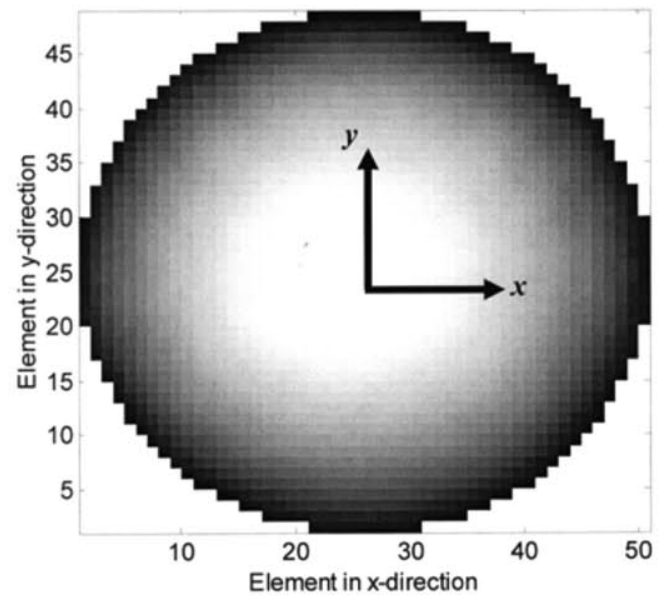

(b)

Fig. 5. Phase distribution required at the reflectarray surface. (a) Using a $360^{\circ}$ phase-shift range. (b) Using phase delay $\left(3 \times 360^{\circ}\right)$

than the required phase defined by the phase distribution, an error function is defined and a numerical method (false position, Newton-Raphson) is used to adjust the length of the line until the error is below a threshold. In each iteration, the phase is computed by the analysis technique described previously, which takes into account the incidence angle of the impinging wave, the losses produced in the dielectric layers and the coupling effects between the elements of the array by assuming local periodicity. Using the line lengths obtained in the design process, an Autocad command file is generated to produce the mask layout.

The design technique has been applied to one linear polarization but it is easy to achieve two linear polarizations if the position of the slots and the lines are redefined to allow enough room for a second orthogonal slot and line.

\section{RESULTS}

Two reflectarrays with the same dimensions have been designed using the previously described technique to implement the phase distributions shown in Fig. 5. One quarter of the masks for the layer containing the delay lines are shown in Fig. 6 for both cases, truncated phase distribution and TTD distribution. In the first case, the printed lines are reduced with every phase

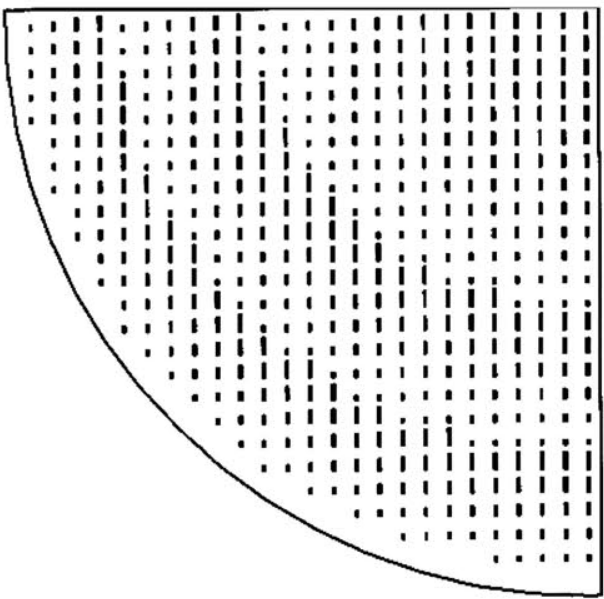

(a)

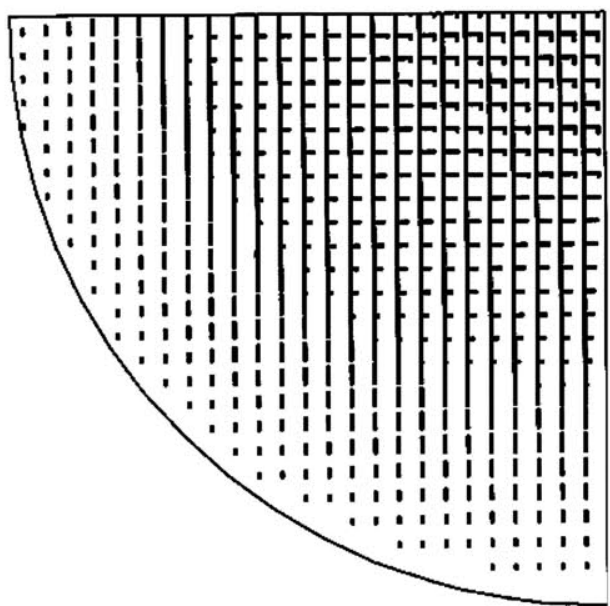

(b)

Fig. 6. One quarter of the masks layout for the layer containing the delay lines. (a) Truncated phase distribution. (b) True-time delay distribution.

jump while in the second case the line length increases without restriction, forming a U-shaped line.

The radiation patterns for the designed reflectarrays were computed from the phase and amplitude of the reflected field at each radiating element. The radiation patterns are computed in gain, dividing by the total power radiated by the horn, and taking into account ohmic losses, losses produced by phase errors and spillover. The 2-D gain patterns for the TTD at $9.65 \mathrm{GHz}$ are shown respectively for the copolar and cross-polar components in Fig. 7.

In order to compare the reflectarray behavior using the two phase distributions, Figs. 8 and 9 show, respectively, the radiation patterns in $u$ and $\mathrm{v}$ coordinates $(u=\sin \theta \cos \varphi, v=$ $\sin \theta \sin \varphi$ ), for both cases, truncated and unlimited phase distributions at central frequency. In both figures, copolar and crosspolar components are shown. As can be seen, the pattern is practically the same for the two phase distributions at the central frequency. A pencil beam with a maximum gain of $36.3 \mathrm{~dB}$ has been obtained with a cross-polar radiation $30 \mathrm{~dB}$ below the maximum gain, which is produced by the square patches. For frequencies above and below $9.65 \mathrm{GHz}$, the beam distortion is larger for the reflectarray with phase-shift limited to $360^{\circ}$. This 


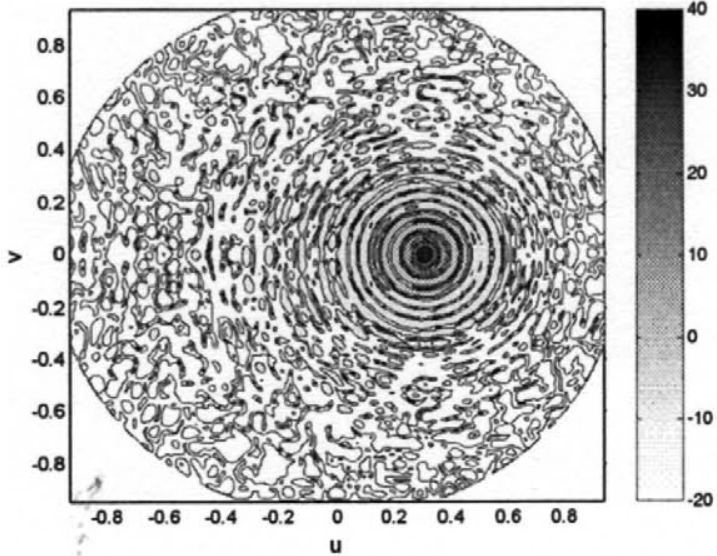

(a)

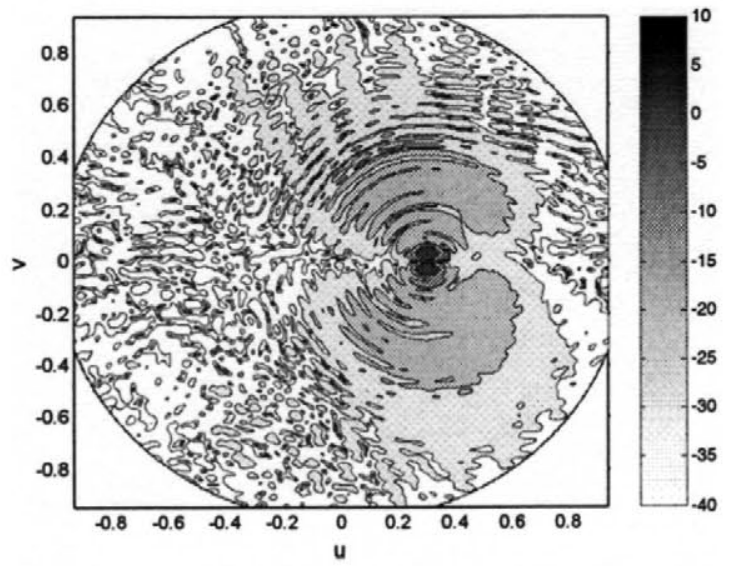

(b)

Fig. 7. Radiation patterns for the TTD reflectarray at $9.65 \mathrm{GHz}$. (a) Copolar (b) Cross-polar.

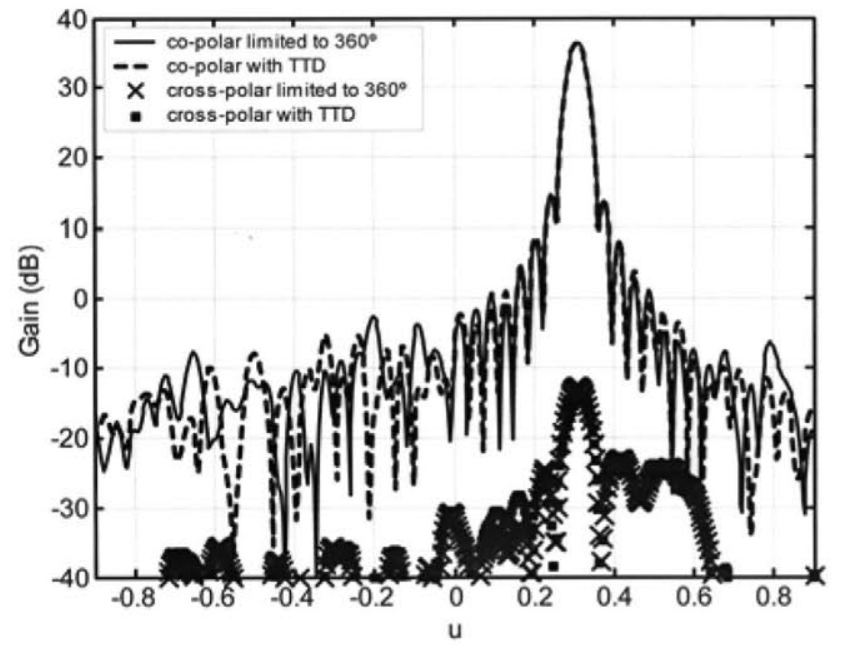

Fig. 8. Radiation pattern comparison in the $X Z$ plane $(v=0)$ at central frequency $(9.65 \mathrm{GHz})$.

can be seen in Figs. 10 and 11 at $8.65 \mathrm{GHz}$, and in Figs. 12 and 13 at $10.65 \mathrm{GHz}$. In the $8.65-\mathrm{GHz}$ case, a sidelobe around $19 \mathrm{~dB}$ is overlapping with the main beam for the truncated phase pattern, while the TTD pattern basically conserves its form and only a small sidelobe appears for the $\mathrm{u}$ plane $20 \mathrm{~dB}$ below the maximum. In the $10.65-\mathrm{GHz}$ case, a beam squint appears in the u plane for the reflectarray with phase limitation. This is mainly

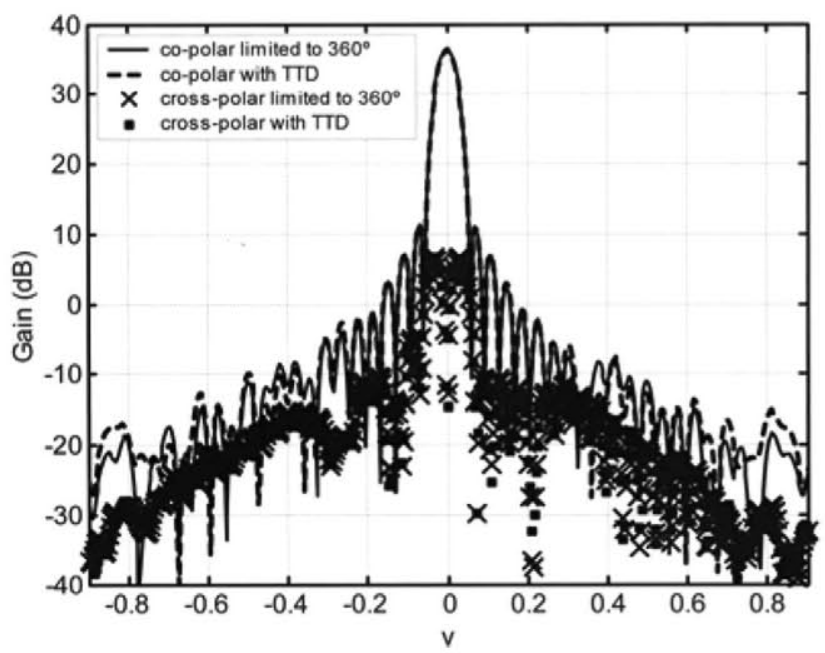

Fig. 9. Radiation pattern comparison in the plane $u=\sin \left(18^{\circ}\right)$ at central frequency $(9.65 \mathrm{GHz})$.

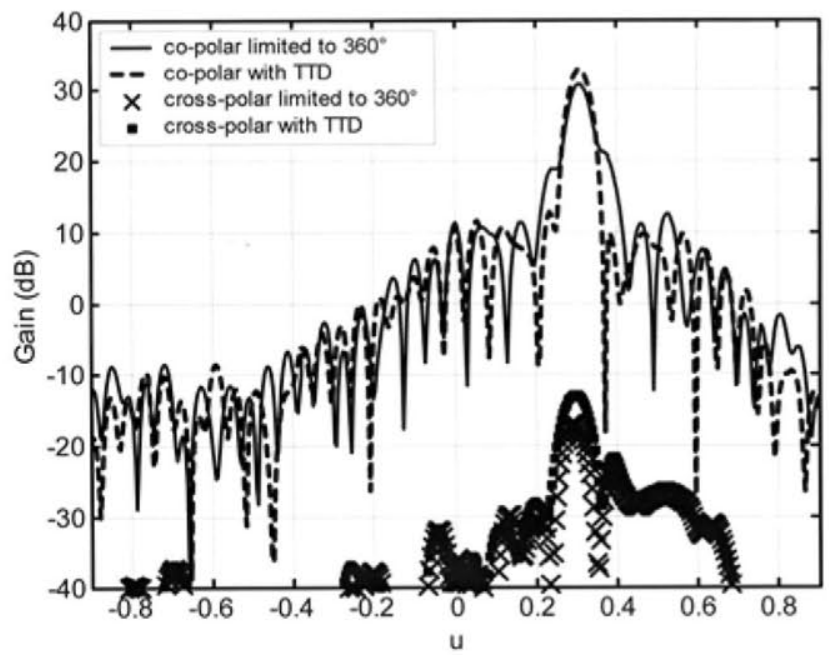

Fig. 10. Radiation pattern comparison in the $X Z$ plane $(v=0)$ at $8.65 \mathrm{GHz}$.

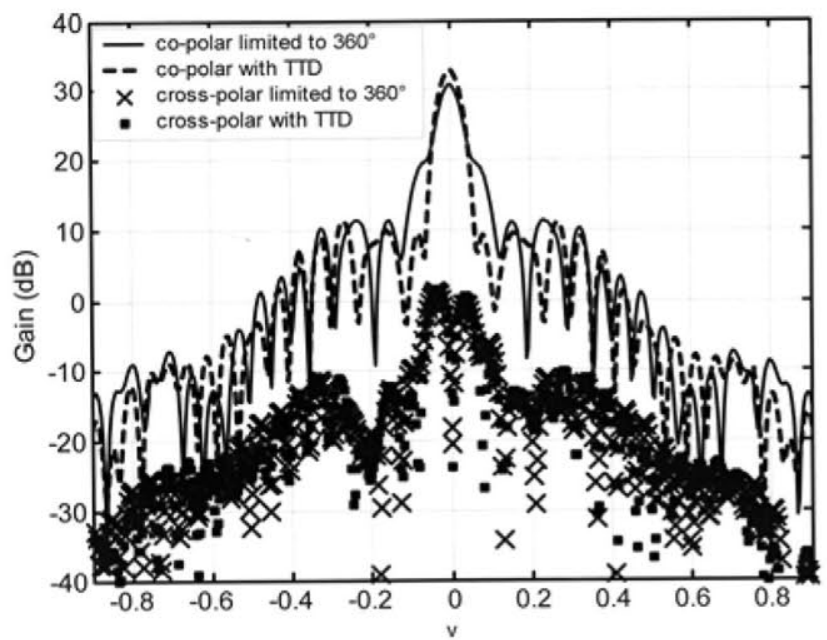

Fig. 11. Radiation pattern comparison in the plane $u=\sin \left(18^{\circ}\right)$ at $8.65 \mathrm{GHz}$.

motivated because in the TTD case, the differential spatial phase delay has been compensated and the only source of phase errors is the nonlinear behavior of the radiating element at extreme frequencies. 


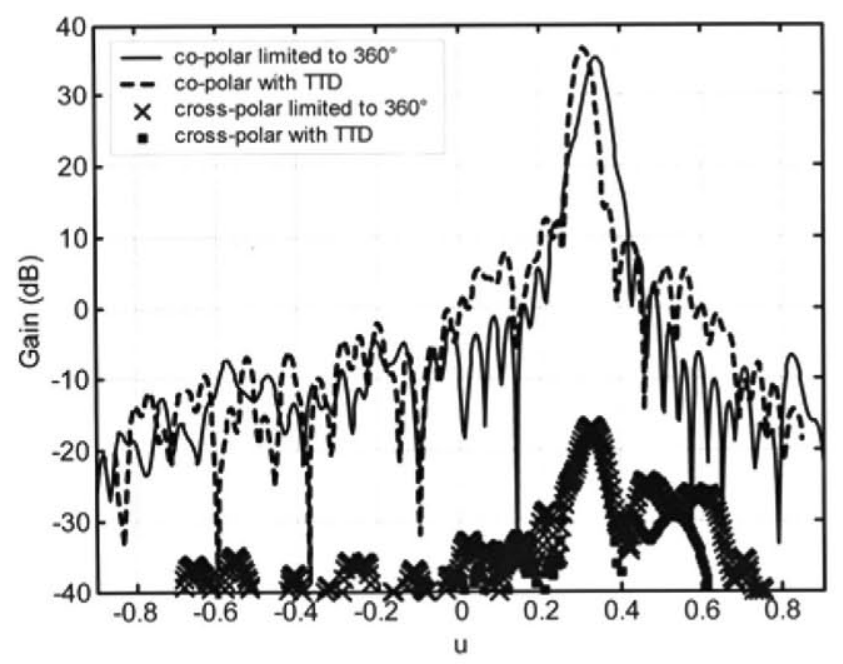

Fig. 12. Radiation pattern comparison in the $X Z$ plane $(v=0)$ at $10.65 \mathrm{GHz}$.

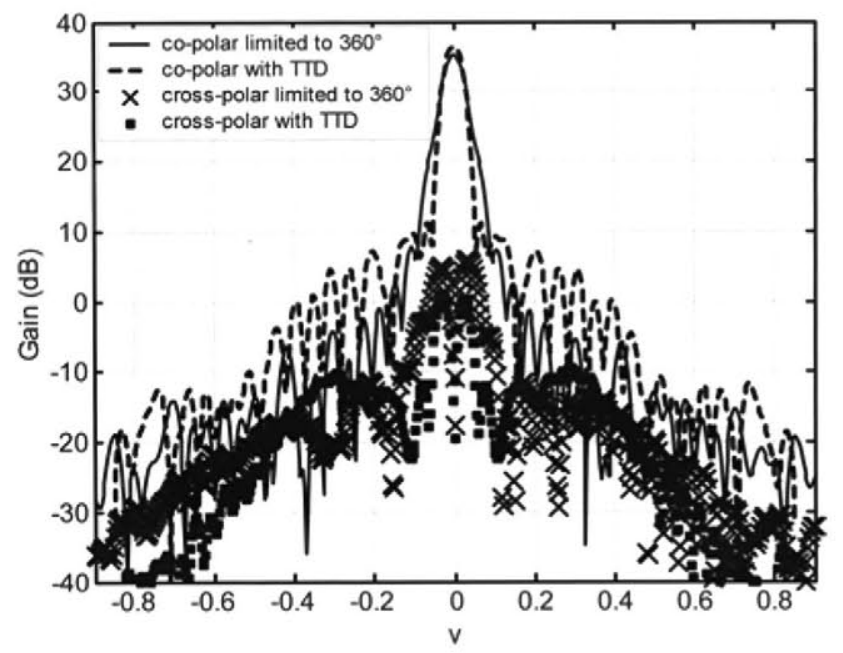

Fig. 13. Radiation pattern comparison in the plane $u=\sin \left(18^{\circ}\right)$ at $10.65 \mathrm{GHz}$

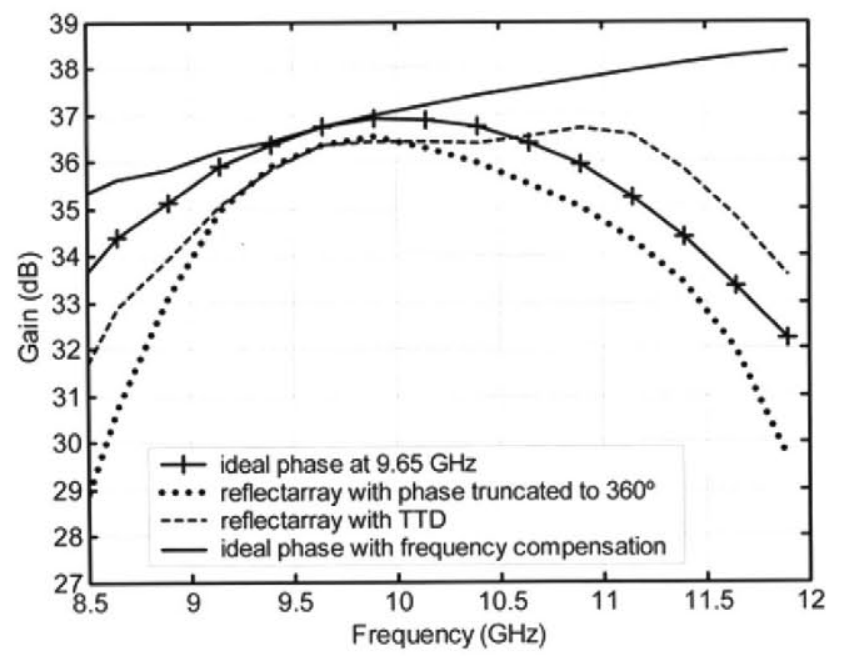

Fig. 14. Gain comparison for the two designed reflectarrays and the gain using ideal phases.

Fig. 14 shows the antenna gain as a function of frequency for the two designed reflectarrays, based on truncated and unlimited

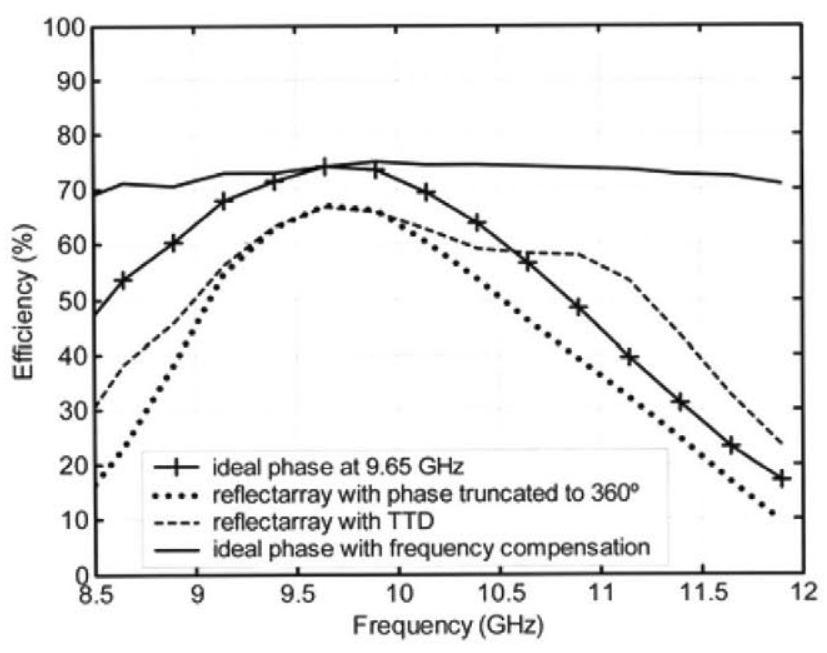

Fig. 15. Efficiency factor for the two designed reflectarrays compared with ideal phases case.

phase distributions. The gain curves corresponding to two theoretical phase distributions have been included, as references. The first ideal curve, represents the gain in the $8.5-11.90 \mathrm{GHz}$ band obtained from the ideal truncated phase distribution at $9.65 \mathrm{GHz}$, shown in Fig. 5(a). This case is equivalent to the design of a reflectarray with $360^{\circ}$ phase truncation, using ideal reflectarray elements that produce the same phase-shift at every frequency. In this case, the phase distribution on the reflectarray remains fixed when frequency varies; however, the required phase to produce a focused beam should vary with frequency proportionally to the true-time delay, as in the case of parabolic reflectors. The second ideal curve represents the gain at each frequency corresponding to the ideal phase distribution, compensated using TTD. Therefore, both ideal curves coincide at the central frequency and their difference represents the diminution of gain produced by the effect of differential phase delay in reflectarrays. Note that the behavior of the gain for the reflectarray with truncated phases is similar as the one of the ideal phase at $9.65 \mathrm{GHz}$, being the reduction in gain for the reflectarray mainly produced by the losses in the dielectric materials $(0.5 \mathrm{~dB})$ and by some distortion of the element phasing at extreme frequencies, as can be seen in Figs. 3(a) and 4(a). On the other hand, the gain curve of the TTD reflectarray should be similar as the one obtained for the ideal compensated phase distribution, which is truth from 9.2 to $10.8 \mathrm{GHz}$. In this frequency range, the TTD curve is similar to the ideal case with a reduction in gain less than $1 \mathrm{~dB}$, as a result of ohmic losses and small phase errors out of central frequency. For extreme frequencies, the gain drops because the phasing produced by the elements are much different that the ideal case, as shown in Figs. 3(a) and 4(a). These curves show clearly that the introduction of TTD lines significantly increases the bandwidth of the reflectarray. For example, the bandwidth for a $0.3-\mathrm{dB}$ gain variation is $10.1 \%$ for the reflectarray with truncated phase and $20.0 \%$ for the TTD reflectarray. If the gain variation is limited to $1.5 \mathrm{~dB}$ below the maximum, the bandwidth increases from $17.8 \%$ to $26.7 \%$ for the TTD reflectarray.

Fig. 15 shows the antenna efficiency expressed as the ratio of the gain and the theoretical directivity of an aperture. As 


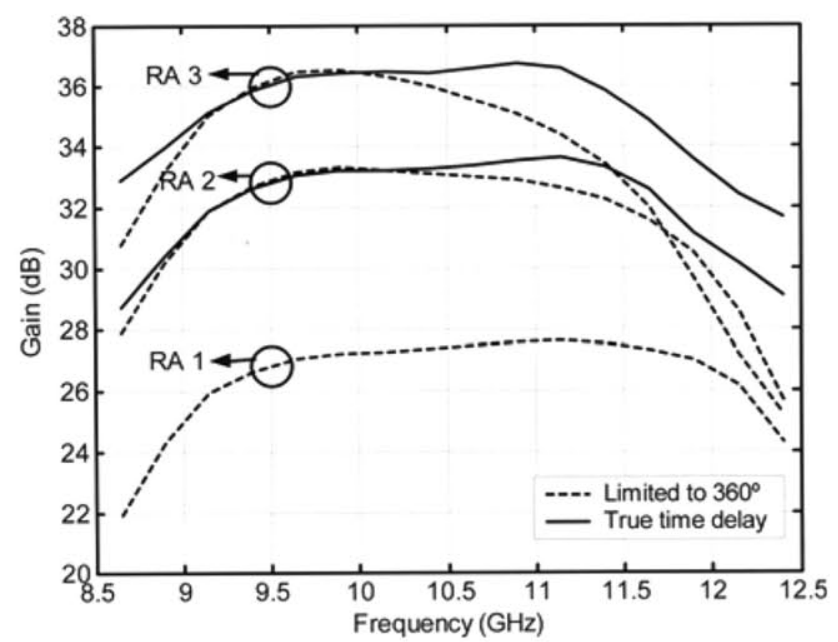

Fig. 16. Gain comparison for different reflectarrays size.

TABLE III

REFLECTARRAY FEATURES

\begin{tabular}{cccc} 
Reflectarray & Physical Size & Electrical Size & Required phase \\
\hline \hline RA 1 & $280 \mathrm{~mm}$ & $9 \lambda$ & 1 cycle \\
\hline RA 2 & $560 \mathrm{~mm}$ & $18 \lambda$ & 2 cycles \\
\hline RA 3 & $840 \mathrm{~mm}$ & $27 \lambda$ & 3 cycles
\end{tabular}

can be seen, the designed reflectarrays allow $60 \%$ efficiency for the working band. When the frequency is varied, the efficiency for the TTD reflectarray remains at least $10 \%$ over the efficiency achieved by a phase distribution limited to one $360^{\circ}$ cycle. The antenna efficiency shown in Fig. 15 for the designed reflectarrays takes into account ohmic losses, losses produced by phase errors, spillover and antenna illumination. The antenna efficiency for the ideal phase distribution, which is above $70 \%$, only includes spillover and antenna illumination.

The effect of different path lengths is only significant for very large electrical dimensions, as in the case of antennas for space applications. With the purpose of obtaining a bandwidth curve as a function of the reflectarray size, different reflectarrays have been designed to generate a pencil beam in the same direction. The reflectarrays have been designed from the smallest case when only one $360^{\circ}$ cycle is required to the $3 \times 360^{\circ}$ previously designed case. The bandwidth decreases as the reflectarray size increases, as expected. This decrease is more important when the phase distribution is limited to $360^{\circ}$. Fig. 16 shows the gain for the three reflectarrays described in Table III. As can be seen, the gain provided by the RA 1 provides a highest bandwidth because only one $360^{\circ}$ cycle is required at the phase distribution and the differential spatial phase delay does not exist. Fig. 17 shows the relative bandwidth in percentage for a variation in gain of $0.3 \mathrm{~dB}$, as a function of the reflectarray electrical size for both cases: phase distribution is limited to $360^{\circ}$ and TTD. The bandwidth of a reflectarray with phase distribution limited to $360^{\circ}$ behaves in a similar way to that of a zoned lens antenna. Fig. 18 shows the bandwidth for a reduction in gain of $1.5 \mathrm{~dB}$. As can be seen in both figures, the errors produced by the nonconstant path from the feed horn to each element of the reflectarray are more important in large reflectar-

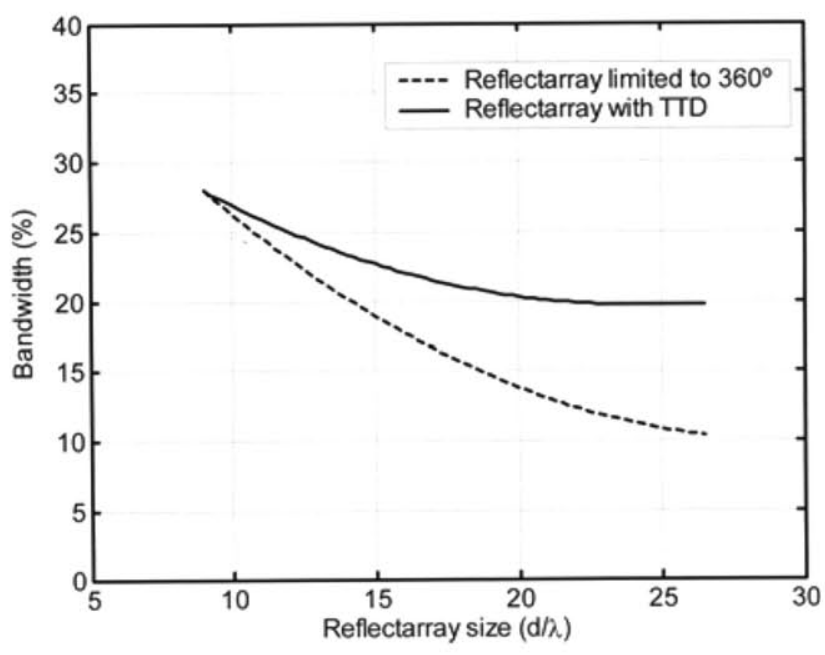

Fig. 17. Bandwidth comparison, at $0.3 \mathrm{~dB}$, between a phase-shift limited to $360^{\circ}$ and TTD for different reflectarray sizes.

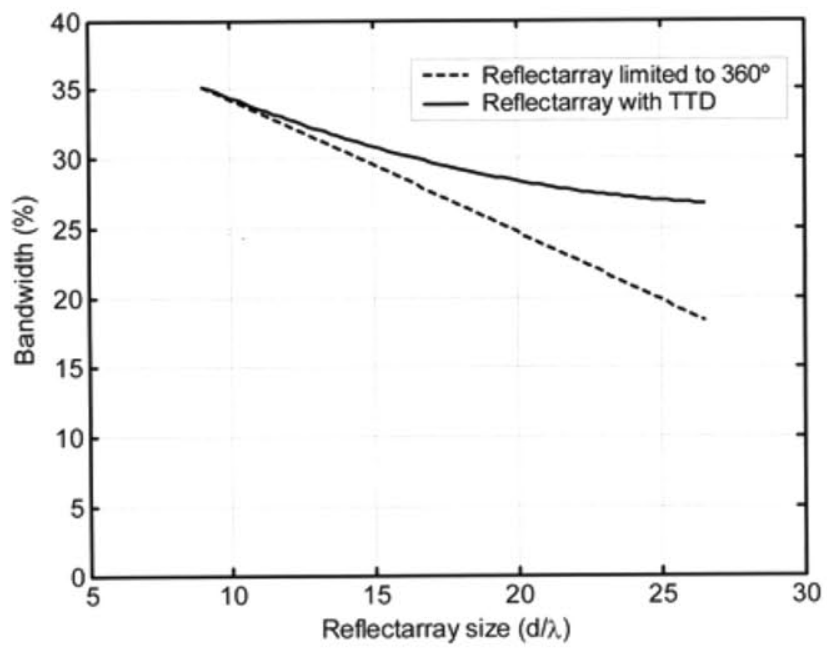

Fig. 18. Bandwidth comparison, at $1.5 \mathrm{~dB}$, between a phase-shift limited to $360^{\circ}$ and TTD for different reflectarray sizes.

rays and can be compensated through the introduction of TTD lines. Figs. 17 and 18 show that, in conventional reflectarrays, the bandwidth decreases as the electrical size of the antenna increases. On the other hand, the relative bandwidth in the case of TTD converges asymptotically for large reflectarrays, because bandwidth is only limited by the reflectarray element and not by its size. These results show that a $20 \%$ bandwidth can be achieved for reflectarrays with aperture dimensions larger than 25 wavelengths.

\section{CONCLUSION}

Several reflectarray antennas have been designed in the $\mathrm{X}$-band, considering two cases for the phase distribution. In the first case, the phase distribution has been truncated to $360^{\circ}$ and in the second case the antennas have been designed without phase limitation using TTD. A significant improvement in bandwidth has been demonstrated when TTD is used where the only limitation is imposed by the bandwidth of the radiating element. The effect of the differential spatial phase delay is 
more important in large reflectarrays as has been demonstrated. This type of antenna can be used in beam scanning applications, if some switches are included in the delay lines.

\section{REFERENCES}

[1] J. Huang, "Bandwidth study of microstrip reflectarray and a novel phased reflectarray concept," in Proc. IEEE Int. Symp. Antennas Propag., Newport Beach, CA, Jun. 1995, pp. 582-585.

[2] D. M. Pozar, "Bandwidth of reflectarrays," Electron. Lett., vol. 39, no. 21, pp. 1490-1491, Oct. 2003.

[3] J. A. Encinar, "Design of two-layer printed reflectarrays using patches of variable size," IEEE Trans. Antennas Propag., vol. 49, no. 10, pp. 1403-1410, Oct. 2001.

[4] J. A. Encinar and J. A. Zornoza, "Three-Layer printed reflectarrays for contoured beam space applications," IEEE Trans. Antennas Propag., vol. 52, no. 5, pp. 1138-1148, May 2004.

[5] N. Misran, R. Cahill, and V. Fusco, "Design optimisation of ring elements for broadband reflectarray antennas," Proc. Inst. Elect. Eng., Microw., Antennas, Propag., vol. 150, no. 6, pp. 440-444, Dec. 2003.

[6] M. R. Chaharmir, J. Shaker, M. Cuhaci, and A. Ittipiboon, "Broadband reflectarray antenna with double cross loops," Electron. Lett., vol. 42, no. 2 , pp. $65-66$, Jan. 2006

[7] M. Bozzi, S. Germani, and L. Perregrini, "Performance comparison of different element shapes used in printed reflectarrays," Ante. Wireless Propag. Lett., vol. 2, no. 1, pp. 219-222, 2003.

[8] D. M. Pozar, "Wideband reflectarrays using artificial impedance surfaces," Electron. Lett., vol. 43, no. 3, pp. 148-149, Feb. 2007.

[9] J. A. Encinar and J. A. Zornoza, "Broadband design of three-layer printed reflectarrays," IEEE Trans. Antennas Propag., vol. 51, no. 7, pp. 1662-1664, Jul. 2003.

[10] A. Bhattacharyya, "Slot-coupled patch reflect array element for enhanced gain-bandwitdh performance," U.S. 6388 620, 2000.

[11] M. E. Bialkowski and H. J. Song, "Dual linearly polarized reflectarray using aperture coupled microstrip patches," in Proc. Antennas Propag. Soc. Int. Symp., Jul. 2001, pp. 486-489.

[12] S. M. Duffy and S. D. Targonski, "Comparison of two flat reflector-type designs for dual-polarization, dual-band operation," in Proc. Antennas Propag. Soc. Int. Symp., Jul. 2001, pp. 288-291.

[13] T. A. Milligan, Modern Antenna Design, 2nd ed. Piscataway, NJ: IEEE Press, 2005, pp. 447-473.

[14] D. G. Bodnar, "Lens antennas," in The Handbook of Antenna Design. London, U.K.: Peter Peregrinus, 1986, vol. 1 and 2, ch. 16, pp. 1-31.

[15] D. C. Chang and M. C. Huang, "Microstrip reflectarray antenna with offset feed," Electron. Lett., vol. 28, no. 16, pp. 1489-1491, 1992.

[16] E. Carrasco, B. Alfageme, and J. A. Encinar, "Design of a multilayer aperture-coupled cell used as phase-shifter in reflectarrays," J. Int. Nice Antennes, pp. 194-195, 2004.

[17] E. Carrasco, M. Barba, and J. A. Encinar, "Reflectarray element based on aperture-coupled patches with slots and lines of variable length," IEEE Trans. Antennas Propag., vol. 55, no. 3, pp. 820-825, Mar. 2007.

[18] C. H. Wan and J. A. Encinar, "Efficient computation of generalized scattering matrix for analyzing multilayer periodic structures," IEEE Trans. Antennas Propag., vol. 43, no. 11, pp. 1233-1242, Nov. 1995.

[19] E. Carrasco, M. Barba, and J. A. Encinar, "Aperture-coupled reflectarray element with wide range of phase delay," Electron. Lett., vol. 42 , no. 12 , pp. $667-668,2006$.

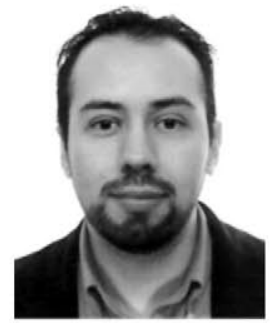

Eduardo Carrasco ( $\left.\mathrm{S}^{\prime} 96\right)$ was born in Mexico City, Mexico. He received the Telecommunication Engineer degree from the Universidad Nacional $\mathrm{Au}$ tonoma de Mexico (UNAM), Mexico City, in 2000. $\mathrm{He}$ is currently working toward the $\mathrm{Ph} . \mathrm{D}$. degree at the Universidad Politécnica de Madrid, Spain.

From 1999 to 2001 he was with Directv Latin America as a Broadcast Operation System Specialist. In 2002 he received a grant from Fundacion Vodafone, Spain, to obtain a Telecommunication Management Degree at the Escuela de Organizacion Industrial (EOI), Spain. Since 2003 he has been with the Electromagnetism and Circuits Theory Department, Universidad Politécnica de Madrid, Spain. From January to April 2008, he visited the Microwave Engineering Laboratory, Università degli Studi di Perugia, Italy. His research interests include printed reflectarrays, multilayer periodic structures, and space antennas.

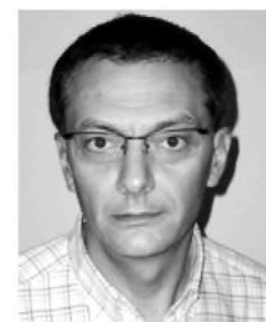

José A. Encinar (S'81-M'86) was born in Madrid, Spain. He received the Electrical Engineer and $\mathrm{Ph} . \mathrm{D}$. degrees from the Universidad Politécnica de Madrid (UPM), in 1979 and 1985, respectively.

Since January 1980 has been with the Applied Electromagnetism and Microwaves Group at UPM, as a Teaching and Research Assistant from 1980 to 1982, as an Assistant Professor from 1983 to 1986, and as Associate Professor from 1986 to 1991. From February to October of 1987 he stayed at Polytechnic University, Brooklyn, NY, as a Postdoctoral Fellow of the NATO Science Program. Since 1991 he has been a Professor of the Electromagnetism and Circuit Theory Department at UPM. In 1996 he was with the Laboratory of Electromagnetics and Acoustics at teh Ecole Polytechnique Fédérale de Lausanne, Switzerland, as Visiting Professor. His research interests include numerical techniques for the analysis of multilayer periodic structures, design of frequency-selective surfaces, printed arrays, and reflectarrays. He has published more than 100 journal and conference papers, and he is holder of three patents on array and reflectarray antennas.

Prof. Encinar was a corecipient of the 2005 H. A. Wheeler Applications Prize Paper Award and the 2007 S. A. Schelkunoff Transactions Prize Paper Award, given by IEEE Antennas and Propagation Society.

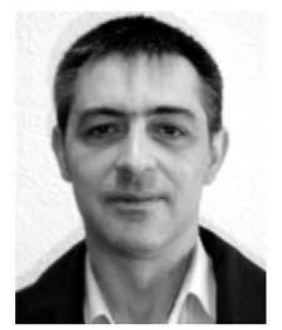

Mariano Barba was born in Murcia, Spain. He received the Ingeniero de Telecomunicación and Ph.D. degrees from the Universidad Politécnica de Madrid (UPM), Spain, in 1990 and 1996, respectively.

From 1991 to 1994 he was with the Departamento de Electromagnetismo y Teoría de Circuitos at UPM, as a Postgraduate Researcher. During this time he developed numerical methods and algorithms to characterize and design microwave passive devices. From 1994 to 2003 he was involved in the R\&D departments of several companies and institutions developing antennas and microwave circuits for space and terrestrial communication applications. In 2003 he rejoined the Departamento de Electromagnetismo y Teoría de Circuitos as an Associate Professor. His research interests include the analysis, characterization, and design of antenna and microwave circuits. He is currently involved in the development and design of multibeam and reconfigurable antennas. 